\title{
A SUFFICIENT CONDITION FOR THE WOLD-CRAMÉR CONCORDANCE OF BANACH-SPACE-VALUED STATIONARY PROCESSES
}

\author{
Grażyna Hajduk-Chmielewska
}

\begin{abstract}
A recent result of Makagon and Salehi [7] is applied to obtain a sufficient condition for the concordance of the Wold decomposition and the spectral measure decomposition of Banachspace-valued stationary processes.
\end{abstract}

A sufficient condition for the Wold-Cramér concordance of Banach-spacevalued stationary processes based on the isomorphism theorem (cf. [8, Theorem 3.3]) is presented in [4]. But for a given stationary process no algorithm for the determination of its isomorphism image is known. The concordance theorem for a stationary process with multiplicity one is given (cf. Proposition 7 below) in a recent paper of Makagon and Salehi [7]. If we connect the technics applied in [4] with the result of [7], we get the sufficient condition for the Wold-Cramér concordance, but for initial Banach-space-valued stationary process is formulated below.

In this paper, $N, Z$ and $K$ stand for positive integers, all integers and the unit circle of the complex plane, respectively. By $\mathcal{B}(K)$ we denote the family of Borel subsets of $K$ and by $m$ the normed Lebesgue measure on $K$. Let $B$ be a complex Banach space with the dual space $B^{*}$ and $H$ be a complex Hilbert space with the inner product $(\cdot, \cdot)$ and the norm $\|\cdot\|$. We denote by $L(B, H)$ the space of all continuous linear operators from $B$ into $H$ and by $\bar{L}^{+}\left(B, B^{*}\right)$ the space of all continuous antilinear and nonnegative operators from $B$ into $B^{*}$.

By a second order stochastic process with values in $B$ we mean a mapping $X: Z \rightarrow L(B, H) . X$ is stationary if its correlation function $R(l, k)=X^{*}(k) X(l)$ depends only on $l-k$. In that case $R$ has the spectral representation

$$
X^{*}(k) X(l)=R(l-k)=\int_{K} z^{l-k} F(d z),
$$

where $F$ is a weakly countably additive measure on $\mathcal{B}(K)$ with values in $\bar{L}^{+}\left(B, B^{*}\right)$. $F$ is called the spectral measure of the process $X$.

We denote $M(X)=\overline{\mathrm{sp}}\{X(l) b: l \in Z, b \in B\}$,

$$
M_{k}(X)=\overline{\operatorname{sp}}\{X(l) b: l \leq k, b \in B\}, \quad M_{-\infty}(X)=\bigcap_{k \in Z} M_{k}(X) .
$$


We say that a stationary process $X$ is separable if $M(X)$ is a separable subspace in $H . X$ is regular if $M_{-\infty}(X)=\{0\}$ and it is singular if $M_{-\infty}(X)=M(X)$.

A second order process $X$ is stationary if and only if $X$ has the unitary shift operator, i.e, if there exists a unitary operator $U: M(X) \rightarrow M(X)$ such that

$$
U X(k)=X(k+1), \quad k \in Z \text {. }
$$

If $E$ is the spectral measure of the operator $U$, then $E$ and $F$ are related by the formula

$$
F(\Delta)=X^{*}(0) E(\Delta) X(0), \quad \Delta \in \mathcal{B}(K) .
$$

Now we recall the Wold decomposition theorem (cf. [2, Theorem 8.6]).

1. Proposition. Let $X: Z \rightarrow L(B, H)$ be a stationary process with the shift operator $U$. Then there exist two processes $X^{r}$ and $X^{s}$ with the same shift operator $U$ such that

(i) $X(k)=X^{r}(k)+X^{s}(k), k \in Z$,

(ii) $M\left(X^{r}\right)$ and $M\left(X^{s}\right)$ are orthogonal,

(iii) for each $k \in Z, M_{k}\left(X^{r}\right)$ and $M_{k}\left(X^{s}\right)$ are contained in $M_{k}(X)$,

(iv) $X^{r}$ is regular and $X^{s}$ is singular.

The above decomposition is unique. Namely, $X^{s}(k)=P_{-\infty} X(k)$ and $X^{r}(k)=X(k)-X^{s}(k)$, where $P_{-\infty}$ is the orthogonal projection onto $M_{-\infty}(X)$.

In particular, it follows that $M\left(X^{s}\right)=M_{-\infty}(X)$ and $M\left(X^{r}\right)=M(X) \ominus$ $M_{-\infty}(X)$.

The following definition of multiplicity of a stationary process is given in [7].

2. Definition. Let $X: Z \rightarrow L(B, H)$ be a separable stationary process. The smallest number $n \in N \cup\{\infty\}$ such that there exists a sequence $\left\{x_{i}\right\}_{i=1}^{n} \subseteq M(X)$ with the property

$$
M(X)=\overline{\operatorname{sp}}\left\{U^{k} x_{i}: 1 \leq i<n+1, k \in Z\right\}
$$

is called the multiplicity of the process $X$ and denoted by $m(X)$. The inequality $i<n+1$ means $i \leq n$ if $n<\infty$ and $i<\infty$ if $n=\infty$.

We will say that the spectral measure $F$ of a stationary process $X$ is absolutely continuous (or singular) with respect to a nonnegative scalar measure $\mu$ if $(F(\cdot) b)(b)$ is absolutely continuous (or singular) with respect to $\mu$ for all $b \in B$. We will use the notations $F<<\mu, F \perp \mu$, respectively. Note that, for fixed $\Delta \in \mathcal{B}(K),(F(\Delta) b)(b)=0$ for each $b \in B$ if and only if $F(\Delta)=0$. Hence $F<<\mu$ is equivalent to the implication that if $\mu(\Delta)=0$, then $F(\Delta)=0$, $\Delta \in \mathcal{B}(K)$.

The following fact is proved in [1, Section 95] and [5, Section 66]. 
3. Proposition. Let $E$ be a spectral measure (on $\mathcal{B}(K))$ in a Hilbert space $H$ and $H^{a}=\{h \in H:(E(\cdot) h, h)<<m\}, H^{s}=\{h \in H:(E(\cdot) h, h) \perp m\}$. Then $H^{a}$ and $H^{s}$ are closed linear subspaces of $H, H=H^{a} \oplus H^{s}, H^{a}$ and $H^{s}$ reducing the spectral measure $E$.

In the case where $E$ is the spectral measure of the shift operator $U$ of a stationary process $X$ and $H=M(X)$, we shall denote $M^{a}(X)=H^{a}, M^{s}(X)=$ $H^{s}$.

4. Lemma. Suppose $X, Y: Z \rightarrow L(B, H)$ are stationary processes with the same shift operator $U$ and $M(Y) \subseteq M(X)$. Denote by $F_{Y}$ the spectral measure of the process $Y$. Then

(i) $F_{Y}<<m$ if and only if, for each $b \in B, Y(0) b \in M^{a}(X)$ (equivalently $\left.M(Y) \subseteq M^{a}(X)\right)$

(ii) $F_{Y} \perp m$ if and only if, for each $b \in B, Y(0) b \in M^{s}(X)$ (equivalently $M(Y) \subseteq$ $\left.M^{s}(X)\right)$.

Proof. In $(\alpha), \quad F_{Y}(\Delta)=Y^{*}(0) E(\Delta) Y(0) ;$ hence $\left(F_{Y}(\Delta) b\right)(b)=$ $(E(\Delta) Y(0) b, Y(0) b)$. Thus, by Proposition $3,\left(F_{Y}(\cdot) b\right)(b)<<m$ if and only if $Y(0) b \in M^{a}(X)$. Furthermore, $Y(0) b \in M^{a}(X)$ for each $b \in B$ implies $M(Y) \subseteq M^{a}(X)$ because $M^{a}(X)$ reduces the shift operator $U$ and $Y(k) b=$ $U^{k} Y(0) b \in M^{a}(X)$. We prove (ii) in the same way.

5. Lemma. Let $F$ be the spectral measure of a stationary process $X$. Then there exists a unique decomposition $F=F^{a}+F^{s}$, where $F^{a}$ and $F^{s}$ are measures with values in $\bar{L}^{+}\left(B, B^{*}\right)$ such that $F^{a}<<m, F^{s} \perp m$.

Proof. We denote $X^{1}(k)=P_{a} X(k), X^{2}(k)=P_{s} X(k)$, where $P_{a}, P_{s}$ are the orthogonal projections on $M^{a}(X), M^{s}(X)$, respectively. Since $M^{a}(X), M^{s}(X)$ reduce the shift operator $U$, both $X^{1}$ and $X^{2}$ are stationary processes with the same shift operator $U$. Let $F^{a}$ be a spectral measure of $X^{1}$ and $F^{s}$ the spectral measure of $X^{2}$. By Lemma $4, F^{a}<<m, F^{s} \perp m$. Moreover,

$$
\begin{aligned}
(F(\Delta) b)(b) & =\|E(\Delta) X(0) b\|^{2}=\left\|E(\Delta)\left(X^{1}(0) b+X^{2}(0) b\right)\right\|^{2} \\
& =\left\|E(\Delta) X^{1}(0) b\right\|^{2}+\left\|E(\Delta) X^{2}(0) b\right\|^{2}=\left(F^{a}(\Delta) b\right)(b)+\left(F^{s}(\Delta) b\right)(b)
\end{aligned}
$$

for each $b \in B$; thus $F=F^{a}+F^{s}$. Next, for each $b \in B,\left(F^{a}(\cdot) b\right)(b)$ is the absolutely continuous and $\left(F^{s}(\cdot) b\right)(b)$ the singular part of the measure $(F(\cdot) b)(b)$ with respect to $m$. The uniqueness of such decomposition implies the uniqueness of the decomposition of $F=F^{a}+F^{s}$.

By [6, Theorem 21.13], it follows that in $M(X)$ there exists a subset $\left\{x_{\gamma}\right\}_{\gamma \in \Gamma}$ ( $\Gamma$ is an index set) such that

$$
M(X)=\bigoplus_{\gamma \in \Gamma} \overline{\operatorname{sp}}\left\{U^{k} x_{\gamma}: k \in Z\right\},
$$


where $U$ is the shift operator of a stationary process $X$. Now fix such decomposition $(\beta)$ and denote by $P_{\gamma}$ the orthogonal projection on $\overline{\mathrm{sp}}\left\{U^{k} x_{\gamma}: k \in Z\right\}$. Let $X_{\gamma}(k)=P_{\gamma} X(k)$. We claim that $X_{\gamma}: Z \rightarrow L\left(B, \overline{\operatorname{sp}}\left\{U^{k} x_{\gamma}: k \in Z\right\}\right)$ is a stationary process with the shift operator $U$. Indeed, $\left.\overline{\operatorname{sp}}\left\{U^{k} x_{\gamma}: k \in Z\right\}\right)$ reduces $U$, whence $P_{\gamma}$ and $U$ commute. Then

$$
X_{\gamma}(k+1)=P_{\gamma} X(k+1)=P_{\gamma} U X(k)=U P_{\gamma} X(k)=U X_{\gamma}(k) .
$$

The following lemma gives a connection between the process $X$ and the family of processes $X_{\gamma}$.

\section{Lemma.}

(i) $\overline{\mathrm{sp}}\left\{U^{k} x_{\gamma}: k \in Z\right\}=M\left(X_{\gamma}\right)$.

(ii) $\overline{P_{\gamma} M_{k}(X)}=M_{k}\left(X_{\gamma}\right)$.

(iii) $P_{\gamma} M_{-\infty}(X) \subseteq M_{-\infty}\left(X_{\gamma}\right)$.

(iv) If $X$ is singular, $X_{\gamma}$ is singular for each $\gamma \in \Gamma$.

(v) If for each $\gamma \in \Gamma X_{\gamma}$ is regular, $X$ is regular.

Proof. (i) From the definition of $P_{\gamma}$ it follows that $\overline{\mathrm{sp}}\left\{U^{k} x_{\gamma}: k \in Z\right\}=$ $P_{\gamma} M(X)$. We prove that $P_{\gamma} M(X)=M\left(X_{\gamma}\right)$. In fact,

$$
P_{\gamma} M(X) \subseteq \overline{\mathrm{sp}}\left\{P_{\gamma} X(l) b: l \in Z, b \in B\right\}=\overline{\mathrm{sp}}\left\{X_{\gamma}(l) b: l \in Z, b \in B\right\}=M\left(X_{\gamma}\right) .
$$

On the other hand, $X_{\gamma}(l) b=P_{\gamma} X(l) b \in P_{\gamma} M(X)$ for each $b \in B$ and $P_{\gamma} M(X)$ is a closed linear subspace. Hence $M\left(X_{\gamma}\right) \subseteq P_{\gamma} M(X)$.

(ii) As in (i) we show that $\overline{P_{\gamma} M_{k}(X)}=M_{k}\left(P_{\gamma} X\right)=M_{k}\left(X_{\gamma}\right)$.

(iii) $P_{\gamma} M_{-\infty}(X)=P_{\gamma}\left(\bigcap_{k \in Z} M_{k}(X)\right) \subseteq\left(\bigcap_{k \in Z} P_{\gamma} M_{k}(X)\right)$

$$
\subseteq \bigcap_{k \in Z} \overline{P_{\gamma} M_{k}(X)}=\bigcap_{k \in Z} M_{k}\left(X_{\gamma}\right)=M_{-\infty}\left(X_{\gamma}\right) \text {. }
$$

(iv) By the assumption $M(X)=M_{-\infty}(X)$. From (iii)

$$
M\left(X_{\gamma}\right)=P_{\gamma} M(X)=P_{\gamma} M_{-\infty}(X) \subseteq M_{-\infty}\left(X_{\gamma}\right) .
$$

Hence $M\left(X_{\gamma}\right)=M_{-\infty}\left(X_{\gamma}\right)$ and each process $X_{\gamma}$ is singular.

(v) Since $M(X)=\bigoplus_{\gamma \in \Gamma} P_{\gamma} M(X)$,

$$
M_{-\infty}(X) \subseteq \bigoplus_{\gamma \in \Gamma} P_{\gamma} M_{-\infty}(X) \subseteq \bigoplus_{\gamma \in \Gamma} M_{-\infty}\left(X_{\gamma}\right)=\{0\},
$$

because every process $X_{\gamma}$ is regular by assumption.

By Lemma 6(i) it follows that every process $X_{\gamma}$ has multiplicity one.

Let now $X=X^{r}+X^{s}$ be the Wold decomposition of a process $X$ as in Theorem 1. We denote by $F_{X} r$ the spectral measure of the process $X^{r}$ and by $F_{X} s$ the spectral measure of $X^{s}$. Since $M\left(X^{r}\right)$ and $M\left(X^{s}\right)$ are orthogonal subspaces and reduce the shift operator $U$, we get $F=F_{X} r+F_{X} s$ as in the proof of Lemma 5 .

The following fact is proved in [7, as Corollary 3.8]. 
7. Proposition. If $X$ is a nonsingular stationary process with multiplicity one, $F^{a}$ is the spectral measure of $X^{r}$ and $F^{s}$ is the spectral measure of $X^{s}$.

Now we state the main result of this paper.

8. Theorem. Let $X: Z \rightarrow L(B, H)$ be a stationary process with the shift operator $U$ and the spectral measure $F$. If there exists a decomposition

$$
M(X)=\bigoplus_{\gamma \in \Gamma} \overline{\mathrm{sp}}\left\{U^{k} x_{\gamma}: k \in Z\right\}
$$

such that for each $\gamma \in \Gamma$ the orthogonal projection $P_{\gamma} X$ of the process $X$ on $\overline{\mathrm{sp}}\left\{U^{k} x_{\gamma}: k \in Z\right\}$ is nonsingular, then $F_{X} r=F^{a}, F_{X} s=F^{s}$.

Proof. By virtue of Lemma 4 it suffices to show that $M\left(X^{r}\right) \subseteq M^{a}(X)$ and $M\left(X^{s}\right) \subseteq M^{s}(X)$. The first of these inclusions follows from Lemma 4 because the spectral measure of a regular process is absolutely continuous with respect to $m$ (cf. [2, Theorem 10.2]). We prove that $X^{s}(0) b \in M^{s}(X)$ for each $b \in B$. In fact,

$$
X^{s}(0) b=\sum_{\gamma \in \Gamma} P_{\gamma} X^{s}(0) b, \quad b \in B
$$

and $P_{\gamma} X^{s}(0) b \in P_{\gamma} M\left(X^{s}\right)=P_{\gamma} M_{-\infty}(X) \subseteq M_{-\infty}\left(X_{\gamma}\right)=M\left(X_{\gamma}^{s}\right)$, where the above inclusion follows from Lemma 6(iii). Since, for each $\gamma \in \Gamma, X_{\gamma}$ has multiplicity one, Proposition 7 implies that the spectral measure of $X_{\gamma}^{s}$ is singular with respect to $m$. Moreover, $U$ is the shift operator of $X_{\gamma}$ and $X_{\gamma}^{s}$ and $M\left(X_{\gamma}^{s}\right) \subseteq M\left(X_{\gamma}\right) \subseteq M(X)$ for every $\gamma \in \Gamma$. Thus $X^{s}(0) b \in M^{s}(X)$ for each $b \in B$, which gives $M\left(X^{s}\right) \subseteq M^{s}(X)$.

9. Corollary. If the assumptions of Theorem 8 hold and the spectral measure of $X$ is absolutely continuous with respect to $m$, then $X$ is regular.

10. Remarks. (a) If $X$ is a separable stationary process with multiplicity $m(X)$ there exists in $M(X)$ a sequence $\left\{x_{i}\right\}_{i=1}^{m(X)}$ realizing the decomposition ( $\beta$ ) (cf. [3, p. 914-918] or [7, Lemma 2.2]). In that case the power of this decomposition is minimal. In a general case the power of the set $\Gamma$ depends on the choice of a subset realizing $(\beta)$.

(b) If a Hilbert space $H$ is separable, Proposition 3 obtains a stronger form. Namely, for a spectral measure $E$ in $H$, there exists in this case the unique decomposition $E=E^{a}+E^{s}$, where $E^{a}$ is a spectral measure in a subspace $H^{a} \subseteq H, E^{s}$ is a spectral measure in a subspace $H^{s} \subseteq H, H^{a} \oplus H^{s}=H$ and $E^{a}<<m, E^{s}$ is concentrated on a set of $m$-zero measure (then $E^{a}$ and $E^{s}$ are supported on disjoint Borel subsets of $K$ ). Indeed, if for some orthonormal base $\left\{e_{i}\right\}_{i=1}^{\infty}$ in $H$ we define

$$
\mu(\Delta)=\sum_{i=1}^{\infty}\left(E(\Delta) e_{i}, e_{i}\right) \cdot 2^{-i}, \quad \Delta \in \mathcal{B}(K),
$$


then $\mu$ is a nonnegative finite measure and $E<<\mu$. Consider the Lebesgue decomposition of $\mu$ with respect to $m: \mu=\mu_{a}+\mu_{s}$. Let $\Delta_{a} \in \mathcal{B}(K)$ be a set such that $m\left(\Delta_{a}^{C}\right)=0$ and $\mu_{s}$ is concentrated on $\Delta_{a}^{C}$. If we put $E^{a}(\Delta)=E\left(\Delta_{a} \cap \Delta\right)$, $E^{s}(\Delta)=E\left(\Delta_{a}^{C} \cap \Delta\right), H^{a}=E\left(\Delta_{a}\right) H, H^{s}=E\left(\Delta_{a}^{C}\right) H$, we get the desired decomposition. It is easy to verify that the subspaces $H^{a}$ and $H^{s}$ are the same as in Proposition 3.

(c) Suppose that $X$ is a separable stationary process with the shift operator $U$ and the spectral measure $F$. By (b) it follows that there exists the unique decomposition $F=F^{a}+F^{s}$ where $F^{a}<<m, F^{s}$ is concentrated on a set of $m$-zero measure. In fact, let $E$ be the spectral measure of $U$. The measures $F^{a}(\Delta)=X^{*}(0) E^{a}(\Delta) X(0), F^{s}(\Delta)=X^{*}(0) E^{s}(\Delta) X(0), \Delta \in \mathcal{B}(K)$, satisfy the above condition.

11. Corollary. Let $X: Z \rightarrow L(B, H)$ be a separable stationary process with multiplicity $m(X)$. Let $F$ be the spectral measure of $X$ and $U$ its shift operator. Then

(a) There exists a unique decomposition $F=F^{a}+F^{s}$, where $F^{a}$ and $F^{s}$ are measures with values in $\bar{L}^{+}\left(B, B^{*}\right)$ such that $F^{a}<<m, F^{s}$ is concentrated on set of $m$-zero measure;

(b) if there exists in $M(X)$ a sequence $\left\{x_{i}\right\}_{i=1}^{m(X)}$ such that

$$
M(X)=\bigoplus_{i=1}^{m(X)} \overline{\mathrm{sp}}\left\{U^{k} x_{i}: k \in Z\right\}
$$

and every process $P_{i} X(1 \leq i<m(X)+1)$ is nonsingular, $F^{a}$ is the spectral measure of $X^{r}$ and $F^{s}$ is the spectral measure of $X^{s}$. ( $P_{i}$ denotes the orthogonal projection on $\overline{\operatorname{sp}}\left\{U^{k} x_{i}: k \in Z\right\}, 1 \leq i<m(X)+1$.) 


\section{References}

[1] Achiezer, N.I., and I.M. Glazman: Theory of linear operators in Hilbert space. - Publications of Kharkov University, Kharkov, 1978 (Russian).

[2] Chobanyan, S.A., and A. Weron: Banach-space-valued stationary processes and their linear prediction. - Dissertationes Math. 125, 1975, 1-45.

[3] Dunford, N., and J.T. Schwartz: Linear operators, Part II. - Spectral Theory, Interscience, New York, 1963.

[4] Hajduk-Chmielewska, G.: The Wold-Cramér concordance problem for Banach-spacevalued stationary processes. - Studia Math. 91, 1988, 31-43.

[5] Halmos, P.: Introduction to Hilbert space and the theory of spectral multiplicity. Chelsea Publishing Company, New York, 1951.

[6] Hewitt, E., and K.A. Ross: Abstract harmonic analysis, Part I. - Springer-Verlag, Berlin-Göttingen-Heidelberg, 1963.

[7] MAKAGON, A., and H. SALEHI: Infinite dimensional stationary sequence with multiplicity one. - Ann. Acad. Sci. Fenn. Ser. A I Math. 12, 1987, 135-150.

[8] Weron, A.: Second order stochastic processes and the dilatation theory in Banach space. - Ann. Inst. H. Poincaré 16, 1980, 29-38.

Szczecin Technical University

Institute of Mathematics

Al. Piastów 17

PL-70-310 Szczecin

Poland

Received 15 January 1988 